\title{
Al Professor Riccardo Giorgino
}

\author{
Luigi Laviola ${ }^{1}$
}

Accettato: 14 giugno 2021 / Pubblicato online: 6 agosto 2021

(c) The Author(s), under exclusive licence to Springer Nature Switzerland AG 2021

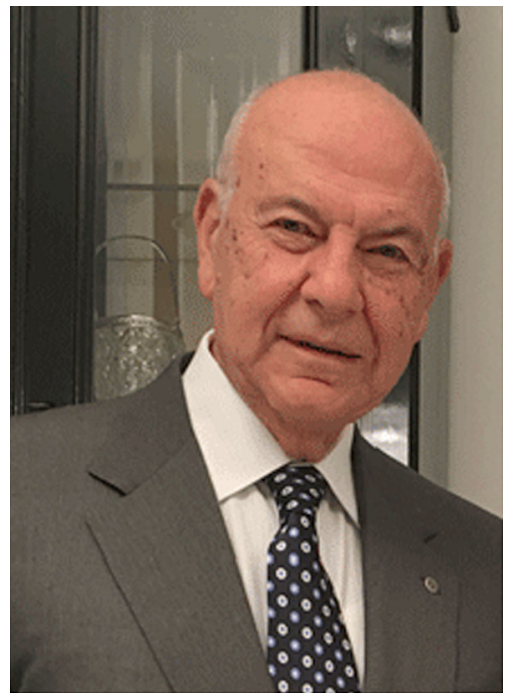

Il Professor Riccardo Giorgino, uno dei padri fondatori dell'Endocrinologia Italiana, ci ha lasciati il 25 maggio 2021. Il 23 giugno avrebbe compiuto 88 anni, 64 dei quali dedicati ai pazienti e agli allievi, come Egli stesso amava dire. Provare a ricordare in queste righe la sua ampia esperienza professionale significa anche ripercorrere un pezzo importante della Endocrinologia italiana e testimoniare la solidità e le profonde radici della comunità endocrinologica e diabetologica pugliese, di cui il Professor Giorgino è stato il Fondatore e il Maestro.

Luigi Laviola a nome degli allievi.

$凶$ L. Laviola

luigi.laviola@uniba.it

1 Sezione di Medicina Interna, Endocrinologia, Andrologia e Malattie Metaboliche, Dipartimento dell'Emergenza e dei Trapianti di Organi, Università degli Studi di Bari "Aldo Moro", Bari, Italia
Nato a Crotone, aveva conseguito nel 1957 la Laurea in Medicina e Chirurgia, presso l'Università degli Studi di Roma "La Sapienza". Si era successivamente specializzato in Medicina Interna presso l'Università degli Studi di Bari “Aldo Moro" nel 1962, in Endocrinologia e Malattie metaboliche presso l'Università degli Studi di Roma "La Sapienza" nel 1967 e in Gerontologia e Geriatria presso l'Università degli Studi di Firenze nel 1968.

Il suo percorso formativo si era arricchito con la frequenza dell'Istituto di Farmacologia dell'Università degli Studi di Parma, con il Professor Vittorio Erspamer, nel 1961, e successivamente, nel 1968-69, dell'Istituto di Endocrinologia della Facoltà medica dell'Università di Marsiglia, sotto la guida del Professor Jean Vague.

Riservato per carattere e per attitudine, non era uso al parlare di sé nella vita ordinaria. Tuttavia, il ricordo dell'esperienza umana e professionale dei suoi anni di formazione compariva a volte nei suoi discorsi, quando rivolgendosi ai suoi allievi faceva riferimento a qualche episodio della sua vita romana o del periodo passato all'estero. Da questi brevi cenni trapelava l'entusiasmo di un ambito culturale che lo aveva sempre appassionato, la fortissima motivazione all'eccellenza, e il senso di profondo rispetto e gratitudine per coloro che gli avevano mostrato la via e che considerava $\mathrm{i}$ suoi maestri.

Tra questi, un posto particolare era riservato al Professor Virgilio Chini, sotto la cui guida aveva mosso i primi passi della sua vita accademica e istituzionale: Assistente volontario, poi incaricato e quindi ordinario, con qualifica di Aiuto dal 1971 al 1980 presso l'Istituto di Clinica Medica dell'Università di Bari. Andava particolarmente orgoglioso del suo ruolo di Aiuto del Professor Chini, che nominava spesso come punto di riferimento fondamentale della sua vita e come esempio di competenza professionale, rigore scientifico e senso istituzionale: un patrimonio di valori 
che rappresentavano per lui un ideale testimone da passare alle generazioni successive di allievi e collaboratori.

L'Università di Bari deve moltissimo al suo costante e infaticabile impegno didattico e scientifico prestato in 40 anni di servizio, con stimolo continuo alla promozione delle attività e della crescita culturale dell'Università. A lui si deve la fondazione dell'Istituto di Clinica Medica, Endocrinologia e Malattie Metaboliche, della Scuola di Specializzazione in Endocrinologia e Malattie del Ricambio e del Corso di Laurea in Dietistica; fondamentale è stato il suo contributo per la nascita della Facoltà di Medicina e Chirurgia dell'Università di Foggia.

La sua attività scientifica, testimoniata da oltre 300 pubblicazioni tra libri di testo, lavori originali, rassegne, ha coperto tutti i settori dell' ambito culturale dell'Endocrinologia e delle Malattie Metaboliche, con particolare riferimento allo studio della malattia diabetica, dell'obesità, dei carcinomi della tiroide, della fisiopatologia del sistema adrenergico e della ipertensione endocrina.

Molte delle sue inesauribili energie sono state dedicate all'attività istituzionale, non solo a livello locale nell'Università di Bari, ma anche nell'ambito di Società Scientifiche Nazionali e Internazionali, in qualità di componente della Commissione Scientifica, componente del Consiglio Direttivo e Presidente Nazionale della Società Italiana di Endocrinologia e della Società Italiana di Diabetologia, e di vice-Presidente del Mediterranean Group for the Study of Diabetes. Non ultima, la pluriennale Direzione della rivista scientifica "Il Diabete", organo ufficiale della Società Italiana di Diabetologia. Alla Società Italiana di Endocrinologia e alla Società Italiana di Diabetologia ha dato un apporto indimenticabile con la realizzazione di iniziative fondamentali che hanno tuttora un seguito, quali la istituzione del Premio SIE, la istituzione della Fondazione Fo.Ri.SID, la creazione di progetti di ricerca societari in ambito diabetologico.

Tutti ricordano la sua capacità di fronteggiare a testa alta qualunque situazione, le sue doti di organizzatore meticoloso e lungimirante e di tessitore di relazioni, con la dichiarata volontà di cercare e trovare, sempre, le soluzioni migliori. Memorabile la sua ironia, in grado di "fotografare", con poche parole, una situazione, una persona, un contesto, e che non risparmiava neanche verso sé stesso, come tutti i grandi: indimenticabile il suo duetto con Renzo Arbore, a conclusione del Congresso della Società italiana di Diabetologia, a Bari, nel 2000.

Ognuno di noi, suoi allievi, conserva però nel cuore una personale antologia di ricordi: la partecipazione a tutti i momenti importanti della vita personale (matrimoni, nascite di figli, lutti); lo sguardo tranquillo e protettivo con cui, seduto nelle ultime file, ha sempre assistito alle presentazioni orali di ciascuno di noi, in ogni occasione congressuale; gli improvvisi silenzi, che nascondevano una commozione intima e trattenuta, nei momenti difficili della vita e nelle celebrazioni dei successi della sua Scuola. Una eredità significativa e impegnativa, che raccogliamo e condividiamo, con le parole con cui il Professor Giorgino concludeva la prefazione di uno dei suoi libri: "Il cammino prosegue, e sono sicuro che, grazie a chi ha raccolto questa eredità, raggiungerà mete più fulgide".

Nota della casa editrice Springer Nature rimane neutrale in riguardo alle rivendicazioni giurisdizionali nelle mappe pubblicate e nelle affiliazioni istituzionali. 